\title{
COMPORTAMIENTO DE LA MACROSOMÍA FETAL EN EL HOSPITAL HIPÓLITO UNANUE DE TACNA 2000 - 2004
}

Responsable: Med. José Ferrer Rojas Miembro: Obst. Elsa Pissano Marabotto

\section{RESUMEN}

Objetivos: Determinar la incidencia de traumatismos asociados al parto y comportamiento de la macrosomía fetal, atendidos es el Hospital Hipólito Unanue de Tacna, durante el quinquenio 2000 2004. Diseño: estudio retropectivo. Participantes: 1,631 casos de macrosomia fetal de un total de partos de 15,182 nacimientos.

Resultados: La incidencia global de Macrosomia asociada a los partos en total fue de $10,74 \%$ de una cantidad de un quinquenio con 15,182 partos en general. Los resultados son los siguientes: Existe una prevalencia de partos con mayor porcentaje de edad, 20 a 24 y 25 a 29 con $27,3 \%$ y $27,2 \%$ respectivamente, la edad de estado civil de la madre se encuentra un $63.8 \%$ de convivientes, grado de instrucción secundaria da un promedio de $60 \%$; madres con R.N. edad gestacional, mayor porcentaje de 3740 semanas con $64,7 \%$ y en cuanto a la preeclampsia el $0,6 \%$, factor $\mathrm{RH}+$ $89,8 \%$ y $0,4 \% \mathrm{RH}(-)$, controles prenatales $93,7 \%$ tuvo controles, partos: $67,2 \%$ espontáneo: $31,8 \%$ termina en cesárea, líquido amniótico el $83,1 \%$ a claro verde oscuro un $11,9 \%$ - Sexo: hombres $62,5 \%$ y mujeres $37,5 \%$, APGAR: $710^{\prime} \operatorname{con} 94,7 \%, 46 \operatorname{con} 4,0 \%$ y 03 con $1,3 \%$, edad gestacional el mayor porcentajes a $40 \%$, R.N. Patológicos con un porcentaje $72,3 \%$; madre con patología con $61,0 \%$ - peso de R.N. Macrosómico con 4000 g. con $86 \%$, con 4,500 49998 en un porcentaje de $11,8 \%$.

\section{ABSTRACT}

Objectives: To determine the incidence of traumatisms associated to the childbirth and behavior of the Fetal, assisted Macrosomia is the Hospital Hipolito Unanue of Tacna, during the Five year period $2000-2004$. Design: I study Retropectivo. Participants: 1,631 cases of Fetal Macrosomia of a total of childbirths of 15,182 births.

Results: The global incidence of Macrosomia associated to the childbirths in total was of $10.74 \%$ of a quantity of a Five year period with 15,182 childbirths in general. The results are the following ones: A prevalencia of childbirths exists with more age percentage 20 at 24 and 25 at 29 with $27.3 \%$ and $27.2 \%$ respectively, the age of civil state of of the Mother he/she is 63.8 convivientes $\%$, grade of secondary instruction he/she gives an average of $60 \%$; Mothers with R.N. age gestacional, bigger percentage of $37-40$ weeks with $64.7 \%$ and as for the Preeclampsia $0.6 \%$, factor $\mathrm{RH}+89.8 \%$ and $0.4 \% \mathrm{RH}(-)$, control prenatal $93.7 \%$ tube controls, childbirths: 67.2 spontaneous $\%: 31.8 \%$ finishes in Caesarean operation, I Liquidate Amniotic $83.1 \%$ to dark green clearing $11.9 \%$ - Sex: men $62.5 \%$ and women $37.5 \%$, APGAR: 7 10 ' with $94.7 \%, 4-6$ with $4.0 \%$ and $0-3$ with $1.3 \%$, age gestacional the biggest percentages to $40 \%$, R.N. Pathological with a percentage $72.3 \%$; Mother with Pathology with $61.0 \%$ - weight of R.N. Macrosomico with $4000 \mathrm{~g}$. with $86 \%$, with $4,500-49998$ in a percentage of $11.8 \%$.

\section{INTRODUCCIÓN}

El American Collage of Obstetricians and Gynaecologist (ACOG), en su último boletín, define a la macrosomía fetal como el peso de nacimiento igual o superior a 4500 gramos; sin embargo, aun existe controversia mundial por la definición más exacta.

Existen quienes apoyan la utilización del percentil 90 del peso fetal para la edad gestacional, y otros un valor neto de peso neonatal mayor a 4 000, 4500 ó 5000 gramos. En el Perú la definición más utilizada en la actualidad es el peso de nacimiento de 4000 gramos y más.
Dentro de los factores asociados a la mayor incidencia que existen en Tacna se señalan: la mayor edad del embarazo, obesidad materna, resistencia a la insulina y el incremento de diabetes gestacional. Asi mismo los R.N. macrosómicos se encuentran en mayor riesgo de distocia de hombros, fractura de clavícula, lesión del plexo braquial y asfixia perinatal.

Considerando las diferencias étnicas que presenta nuestra población en relación con los norteamericanos y europeos, existe una gran diferencia en relación con la incidencia de los partos de macrosómicos, ya que no hay mayor frecuencia en lo referente a los traumatismos. 


\section{MATERIAL Y MÉTODOS}

De un total de 15182 nacimientos atendidos en el Servicio de Maternidad del Hospital Hipólito Unanue de Tacna, durante el quinquenio 2000 2004, se consideraron 1,631 casos de macrosomia fetal. La información fue recolectada retrospectivamente, a través de un registro computacional de los antecedentes maternos, el resultado del parto y la evolución del recién nacido.

Se consideraron los nacimientos de primíperas y multíparas, sin cesárea y con una cesárea anterior, cuyas edades gestacionales eran de 37 semanas o más.

\section{RESULTADOS}

El análisis consideró 1,631 casos de nacimiento macrosómico, de los cuales correspondieron a primíparas un $33,2 \%$ y $66,8 \%$ a multiparas. En el $60 \%$ de los casos el trabajo de parto fue en forma espontánea, el $39,1 \%$ fue inducido y el $0,3 \%$ terminó en cesárea.

El porcentaje de R.N. con peso de 4000 a 4 499 gramos fue de $86 \%$, de 4500 a 4999 gramos fue de $11,8 \%$ y de 5000 gramos y más fue con un porcentaje de $2,2 \%$.

En cuanto a la incidencia de traumatismos asociados al parto tenemos los principales traumas o patologias:

- $\quad$ Anemia con un porcentaje de $37,8 \%$ al $56,3 \%$.

- Tracto urinario que va en el quinquenio materia de estudio del $17,7 \%$ al $28,2 \%$.

- Como 3era incidencia de traumatismo asociado al parto tenemos el desgarro perineal con un promedio de $20,0 \%$ durante el quinquenio.

Al evaluar el riesgo de presentar un traumatismo del parto, los R.N. macrosómicos presentan lo siguiente:

- Líquido Amníotico claro (normal> 83,1\%)

Liquido Amniotico verde $11,9 \%$

Sexo: Hombre $62,5 \%$

Mujer $37,5 \%$

- Puntaje de APGAR

$7-10$ con $99,3 \%$

$4-6$ con $0,4 \%$

$0-3$ con $0,3 \%$

- Patología del recién nacido

Hiperglicemia neonal $42,4 \%$

Traumatismo del nacimiento no grave $21,2 \%$. No patológico (R.N.) 72,3\%.

- Tasa de mortalidad materna, fetal, neonatal y perinatal:
- En este quinquenio encontramos lo siguiente:

.. La tasa de mortalidad fetal durante este quinquenio es de $3,5 \times 1000$ N.V. al 13,6 x 1000 N.V.

La tasa de mortalidad neonatal es mínima.

..La tasa de mortalidad materna: solo se .produce 1 muerte el año 2003.

CUADRO N ${ }^{\circ} 1$ Prevalencia de la Macrosomia Fetal

\begin{tabular}{|c|c|c|c|}
\hline $\begin{array}{c}\text { Años de } \\
\text { estudio }\end{array}$ & $\begin{array}{c}\mathbf{N}^{0} \text { de partos } \\
\text { totales }\end{array}$ & $\begin{array}{c}\mathbf{N}^{0} \text { de partos } \\
\text { macrosómicos }\end{array}$ & $\%$ \\
\hline 2000 & 2478 & 285 & 11,50 \\
\hline 2001 & 2740 & 284 & 10,36 \\
\hline 2002 & 3218 & 333 & 10,35 \\
\hline 2003 & 3239 & 361 & 11,14 \\
\hline 2004 & 3507 & 368 & 10,49 \\
\hline TOTAL & 15182 & 1631 & 53,84 \\
\hline
\end{tabular}

En el presente cuadro se observa que la prevalencia de macrosomia fetal durante los 5 años tiene una incidencia de 10,74\%.

CUADRO № 2 Edad Gestacional en Madres con R.N. Macrosómicos

\begin{tabular}{|l|c|c|c|c|c|c|c|c|c|c|}
\hline \multirow{2}{*}{$\begin{array}{c}\text { Años en } \\
\text { estudio }\end{array}$} & 2000 & \multicolumn{2}{|c|}{2001} & \multicolumn{2}{c|}{2002} & \multicolumn{2}{|c|}{2003} & \multicolumn{2}{|c|}{2004} \\
\cline { 2 - 10 } $\begin{array}{c}\text { Edad } \\
\text { Gestacio- } \\
\text { nal }\end{array}$ & $N^{0}$ & $\%$ & $N^{0}$ & $\%$ & $N^{0}$ & $\%$ & $N^{0}$ & $\%$ & $N^{0}$ & $\%$ \\
\hline $28-36$ & 6 & 2,1 & 3 & 1,1 & 7 & 2,1 & 2 & 0,6 & 0 & 0 \\
\hline $37-40$ & 188 & 66,0 & 168 & 59,2 & 209 & 62,8 & 238 & 65,9 & 257 & 69,8 \\
\hline $41-44$ & 91 & 31,9 & 113 & 39,8 & 117 & 35,1 & 121 & 33,5 & 111 & 30,2 \\
\hline Total & 285 & 100,0 & 284 & 100,0 & 333 & 100,0 & 361 & 100,0 & 368 & 100,0 \\
\hline
\end{tabular}

En los 5 años de estudio, el mayor porcentaje corresponde en las comprendidas entre las $37-40$ semanas por $64,7 \%$, y el menor porcentaje corresponde a las 28 - 36 semanas con promedio de $1,2 \%$.

CUADRO No 3 Terminación del Parto en Madre con R.N. Macrosómicos

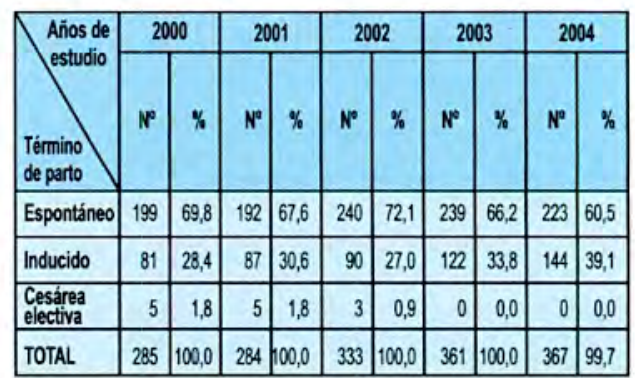

El mayor porcentaje de partos se realiza espontáneamente por vía vaginal con $67.2 \%$, el inducido con $29,9 \%$ y cesárea con $0,9 \%$. 


\section{DISCUSIÓN}

Este estudio estuvo enfocado en la identificación de categoría del R.N. con posible exposición a mayor riesgo de morbilidad asociado al parto de acuerdo con su peso de nacimiento y via de parto.

- Asimismo al evaluar este riesgo dentro de las distintas categorias de macrosomia. Se piensa que el grupo de mayor riesgo está formado por los macrosómicos de gran peso, y es donde deben intensificarse las medidas preventivas y evitar un mal resultado obstétrico.

Los traumatismos más frecuentes observados fueron la fractura de clavícula y cefalohematomas, y solo un porcentaje menor las parálisis braquiales.

Considerando la baja mortalidad materna asociada a la cesárea electiva, de acuerdo con las medidas de asepsia y de control de la postcesárea. Sin embargo, es sumamente difícil realizar en forma prenatal el diagnóstico de macrosomia prenatal debido a que la ecografia presenta de 10 a $15 \%$ de error de estimación. Recientemente RAIO-ET-AL ha planteado que el riesgo de morbilidad fetal no es constante, dependería de la estatura materna, diabetes gestacional, etc.

\section{CONCLUSIONES}

1. El porcentaje de macrosomía fetal en los años 2000 y 2004 es de 10 a $11 \%$.

2. En cuanto a la edad de las pacientes, el mayor porcentaje corresponde al grupo de 20 a 24 años de edad con el $27,3 \%$; el estado civil que prevalece es el de las convivientes con un $63,8 \%$ y en cuanto a los estudios de estas pacientes se observa que el mayor porcentaje corresponde a las que tienen nivel de instrucción secundaria con un $60 \%$.

3. Con respecto a las gestaciones se observa que el mayor porcentaje corresponde a las que han tenido de $1-2$ gestaciones previas con un $49,9 \%$, en cuanto a los partos de igual manera el mayor porcentaje es para las que han tenido de 1-2 partos con un $50 ., 9 \%$; y de acuerdo con la edad gestacional vemos que el mayor porcentaje corresponde a las comprendidas entre las 3740 semanas de gestación con un $64,7 \%$.

4. De acuerdo a los antecedentes personales podemos concluir que las pacientes que tuvieron R.N. Macrosómicos, el $99 \%$ no tuvo el antecedente de diabetes, el $99,7 \%$ no tuvo el antecedente de hipertensión y el $99,4 \%$ no tuvo el antecedente de preeclampsia en el embarazo.

5. Dentro de los 5 años de estudio se observa que el 93,7 tuvo controles prenatales, siendo el inicio del trabajo de parto espontáneo el $79,7 \%$ y la vía del parto en el mayor porcentaje fue el espontáneo con un $67,2 \%$ seguido de la cesárea con el $31,8 \%$.

6. Las características de los recién nacidos macrosómicos fue que el mayor porcentaje con $62,5 \%$ fue de sexo masculino, el apgar al minuto fue de $7-10$ con el $94,7 \%$, el apgar a los 5 minutos de 7-10 con el 9,3\%, la edad gestacional por examen físico fue de $37-40$ semanas en el $92,5 \%$ y el peso prevalente fue de $4000-4499$ grs. con el $86 \%$.

7. Con respecto a la patología de los recién nacidos el $27,6 \%$ tuvo alguna patología, dentro de las cuales las principales fueron las hipoglicemia neonatal con un $42,4 \%$ seguido de las afecciones perinatales con un $21,2 \%$ y traumatismos del nacimientos con $9,1 \%$.

8. En cuanto a la patología de la madre con R.N. macrosómico tenemos que el $61,0 \%$ presenta alguna patología dentro de las cuales la más frecuente es la anemia con un $56,3 \%$ seguido de la infección del tracto urinario con un $24.1 \% \mathrm{y}$, por último, tenemos el desgarro perineal con un $10,2 \%$.

9. Con respecto a la tasa de mortalidad fetal tenemos que en el año 2000 y 2001 esta fue de $3.5 \times 1000$ N.V., incrementándose considerablemente hasta el año 2004 que fue de $13,6 \times 1000$ N.V.. En cuanto a la mortalidad neonatal tenemos que ésta solo se registró en los años 2003 y 2004 con un $2,8 \times 1000$ N.V.. Por lo tanto, la tasa de mortalidad perinatal más alta fue la del año 2004 con un $16,4 \times 1000$ N.V. Con respecto a la mortalidad materna solo se registró un caso en el año 2003.

\section{RECOMENDACIONES}

- Es nuestra obligación realizar un análisis de las posibles causas o antecedentes que pueden condicionar la macrosomía fetal y tomar las correlaciones que están a nuestro alcance para reducirla, actuando sobre los factores clínicos en los cuales podemos influir, como son el embarazo prolongado, la diabetes, la obesidad.

- En cada caso se debe establecer su pronóstico para elegir la vía del parto más adecuada. Consideramos que el parto por cesárea no debe valorarse exclusivamente sobre la base del peso fetal, deben tenerse en cuenta todos los parámetros para decidirlo, y cada departamento de obstetricia deberá plantear una estrategia para cada caso particular.

\section{BIBLIOGRAFÍA}

Hernández S.R. Fernández C. Carlos; Baptista L. Pilar (1997). Métodos de la Investigación Cientifica. Colombia.

Silver, Henry, Kempe, Henry, Bruin, Henry Fulginiti, Vicent. Manual de Pediatria. Edit. México, 1988. 
Schwarcz, Ricardo y Otros. Obstetricia. Editorial ElAteneo, quinta edición. pág 511.

Tizona Rendon, Manuel. Medicina Perinatal, Tacna, 1999, segunda edición. pág 44.

"Macrosomía Fetal" en: Cunnigham, Mac Donaldp P., Gant N., Leyeno K. Gilstrop L.C.
Williams. Obstetricia. 20 Ed. Buenos Aires Edición Panamericana, 1998, pág. 787 - 801

Internet, "Macrosomía . Su comportamiento en el último quinquenio". Cuba, 2002. 